\title{
ARTICLE
}

\section{Cultural factors and sexual dysfunction in clinical practice ${ }^{\dagger}$}

\author{
Vishal Bhavsar \& Dinesh Bhugra
}

\begin{abstract}
Vishal Bhavsar is an Academic Clinical Fellow in the Department of Psychosis Studies, Institute of Psychiatry, King's College London. Dinesh Bhugra is Professor of Mental Health and Cultural Diversity in the Health Service and Population Research Department of the Institute of Psychiatry, King's College London. He is also an honorary consultant at the Maudsley Hospital, where he runs the sexual and couples therapy clinic.

Correspondence Professor Dinesh Bhugra, Health Service and Population Research Department, Institute of Psychiatry, King's College London, De Crespigny Park, London SE5 8AF, UK. Email: dinesh.bhugra@ kcl.ac.uk
\end{abstract}

${ }^{\dagger}$ For a related article, see Bhugra D, Colombini G (2013) Sexual dysfunction: classification and assessment. Advances in Psychiatric Treatment 19: 48-55.

\begin{abstract}
SUMMARY
Attitudes to sex and the perceived role of sexual activity are very strongly influenced by cultural values. Culturally determined gender roles influence relationships between different-sex partners, and cultural values affect attitudes towards sexual variation. Cultures define what is deviant and from where help is sought. Through differing patterns of child-rearing, cultures also affect individuals' cognitive development, world views and explanatory models of emotional distress. It is critical that clinicians are aware of the role of culture in defining sexual dysfunction and how cultural factors can be used in initiating treatment as well as in therapeutic engagement and alliance. Although epidemiological data on prevalence of sexual dysfunction across cultures are scanty, it is likely that prevalences vary, as will pathways into care and patterns of helpseeking. In this article we discuss the potential impact of culture on sexual dysfunction, and issues that clinicians, whether in specialist or in general services, need to be aware of in assessing and treating patients who present with sexual dysfunction.
\end{abstract}

\section{DECLARATION OF INTEREST}

None.

Although the word culture is used broadly, at its core it relates to the shared values and meanings of a particular group. Culture determines and defines our identity. For example, most psychiatrists carry multiple identities related to gender, religion, seniority, specialism and so on, which in clinical practice may manifest in different ways, depending on context. Culture can also be seen in material terms, in other words, as the sum of human productive activity in a given space, including habits, institutions and customs. In broad terms, definitions of culture may be divided into the ecological, referring to the sum of human artefacts of a group, and the cognitive, which refers to the gross network of meanings that link a people together (Barnard 2002). It is important to note the distance between ecological and cognitive theories of culture, which allows a space for what aspects of behaviour, including sexual behaviour, actually signify. Hofstede (2001) suggests that any given culture can be characterised in terms of five dimensions: individualism (egocentric or sociocentric); masculinity or femininity; power distance (the extent to which the less powerful expect and accept that power is distributed unequally); long-term orientation; and toleration of uncertainty. The influence of culture on the development of personality and maintenance of well-being is an important aspect of clinical work with disorders of behaviour, including sexual dysfunction. Medically, sexual dysfunction may be defined as a group of problems occurring in the domains of desire, arousal and orgasm, that may or may not involve pain (Bhugra 2013). We might also add that some sexual dysfunctions are genderspecific, for example vaginismus and ejaculatory and erectile dysfunction, whereas others, such as disorders of desire and orgasm, are diagnosed in both genders.

On this basis, culture is particularly relevant in sexual function and dysfunction, where it is important to understand the way in which behaviour is perceived by the participants. It is inevitable that culture plays a complex role in the production of sexual activity, be it heterosexual, homosexual or alternative sexual minority behaviours. Attitudes to any sexual activity or behaviour are strongly influenced by culture.

\section{Culture and sexual behaviour}

Discussion of the role of culture in determining patterns of sexual behaviour is largely speculative, given the paucity of comparative epidemiological data, especially related to incidence and prevalence, across cultures and across time. Furthermore, the categorisation of sexual conditions and behaviours raises a number of theoretical and semantic difficulties which are described in our previous article (Bhugra 2013). In terms of the level of interest it has garnered by researchers, the study of sexual dysfunction has lost out to gender identity disorders and paraphilias, perhaps because of the perceived 'exotic' nature of these conditions.

For clinicians, the development of coherent models of the relationship between culture and sexual dysfunction, specifically relating to how to 
BOX 1 Aspects of the relationship between culture and sexual dysfunction

- Gender roles: their development and cultural definitions

- Gender-role expectations

- Explanations of sexual behaviour

- Explanatory models of sexual dysfunction

- Personal and cultural beliefs regarding healing

think about the link between the cultural aspects of behaviour and distress, must lie at the heart not only of diagnosis but also of management. The factors listed in Box 1 appear vital in understanding this relationship, particularly in heterosexual couples.

One of the most striking observations regarding sexual dysfunctions of all kinds is the obvious differences in their frequency in different cultural groups (discussed below). This phenomenon lends itself to exploration of how the orientation and configuration of a culture may relate to the causation, manifestation and management of sexual problems.

\section{Assessment and diagnosis}

As with symptoms in any area of medicine, assessment of sexual dysfunction in the clinic does not take place within a contextual vacuum: patients present at particular times, to particular places and clinicians, and for identifiable reasons. Culture patterns the thresholds and expectations by which functioning is evaluated by the individual, their partners and their families as well as by the clinician, the clinical observer. In some cultural settings, the clinical observer of sexual dysfunction is an obstetrician or a primary care physician, in others, a psychiatrist. In many cultures, shamans and alternative practitioners such as hakims play the role of sexologists and sex experts (d'Ardenne 1986).

\section{Ethnocentricity of definitions}

Sexual dysfunction is itself culturally influenced, as cultures create and colour myths about sexuality, masculinity, and sexual prowess and behaviour. Masters $\&$ Johnson's (1970) model of the sexual response cycle, which serves as the theoretical basis of the sensate focus approach to couples sex therapy, is ethnocentric, reflecting an implicit belief in the superiority of American norms of sexual behaviour.

There is thus a cultural influence on the conceptualisation of sexual dysfunction itself which makes a cross-cultural comparison of normative sexual behaviour and sexual dysfunction critical and of interest.

Goettsch (1987) raises important questions about the definition of sexual dysfunction. For example, what renders ejaculation 'premature'? Is it or should it be defined by duration of erection, frequency or any other factors related to satisfaction of the partner? Masters $\&$ Johnson define it as a man reaching orgasm before the partner more than $50 \%$ of the time, without accounting for situations in which the partner does not reach orgasm at all (Griffitt 1985). Reiss (1986) states that among some Melanesians, ejaculation more than $30 \mathrm{~s}$ after insertion is regarded as 'delayed'. One of the important problems here is the sheer number of theoretical frameworks it is possible to apply to the study of human sexuality. Weis (1998) identifies no fewer than 39 separate theoretical frameworks for understanding human sexuality, ranging from evolutionary biology to games theory. Taken alongside the myriad cultural theories used to explain behavioural differences, the literature is complex indeed.

\section{Contexts and concepts}

To understand how culture can and should shape the treatment of individuals and couples with sexual dysfunction, it is necessary to discuss the ways in which cultural issues influence both the way in which sexual dysfunctions are diagnosed and the diagnostic systems themselves. For example, Davis (1998) argues that the idea of an individualised internal sex drive, together with a multistage 'sequence' of sexual intercourse, is a product of a reified and analysable historical process in Western thought. For Davis, Western medical notions of sexual dysfunction are inherently linked to Western biomedical concepts of sexual deviance and 'rooted in the narrowly defined parameters of the human sexual response cycle' - an attitude that might be called 'biological essentialism'. The author points out the paucity of clinical studies on the influence of cultural or social factors on the development of sexual problems, and also notes a predisposition in the literature for 'troublesome ethnic and racial stereotyping'. Davis notes that 'the very implication that human sexuality is a natural, innate drive, an orderly sequence of in-built events that culminate in orgasm that is the same for all times and for all individuals, uninfluenced by class or culture is in itself an artefact of past and present intellectual traditions in Western Culture'.

Gender roles, performance standards and psychological functioning, not to mention the attitudes of clinicians, are all culturally influenced 
(if not determined). Davis (1998) also considers the delicate balances and articulation required to investigate and report potential differences in prevalence rates of dysfunctions in particular groups (she cites Asian males), and the need to avoid singling out particular groups as dysfunctional in a particular aspect.

Cultural beliefs shape not only the prevalence of symptoms, their expression and their exact mode of presentation to professionals, but also the notion of what constitutes a disorder. For example, 'excessive masturbation' is treated as a disorder in some Indian literature (e.g. Singh 1960), and is noted as a presenting problem in an Asian man in a study of sexual dysfunction in Asian couples (Bhugra 1988).

Thus, the concepts of culture, sex, dysfunction and diagnosis are all interrelated. Culture plays a role in defining what is abnormal, what is called abnormal and the underlying cultural patterns.

\section{Cultural epidemiology of sexual dysfunction}

Generalisations regarding variations in prevalence of sexual dysfunction are difficult to make. Population-based studies employing standardised case-finding instruments are outnumbered by smaller studies carried out in clinical settings. The indiscriminate use of instruments developed in one culture to assess people in another carries problems in terms of sensitivity and specificity (Prince 2003).

Epidemiological studies generally attempt 'cultural translation' of measures, most of which are created in the West, for use in other linguistic and national contexts. Methods may involve simple translation into the indigenous language (as in the study by Fatusi et al, 2003), or a more formal process of evaluating the measure through consensus between investigators (as done by Valadares et al, 2008). Developing culturally appropriate constructs for sexual dysfunction is another important endeavour, albeit one that has seen little formal research in epidemiological studies.

\section{The West}

One of the largest prevalence studies of sexual dysfunction in the West in recent times is that of Shifren et al (2008), which measured the prevalence of sexual problems in US women using the Female Sexual Distress Scale, a selfadministered questionnaire on particular aspects of distress related to sex, including frustration, embarrassment and anger. The age-adjusted point prevalence of any female sexual problem was $43.1 \%$, and $22.2 \%$ for sexually related personal distress (defined as a score of at least 15 on the
Female Sexual Distress Scale). A literature review of female sexual dysfunction estimated that, on the basis of previously published literature in the West, $40 \%$ of women in any given country will have some sort of sexual problem, with 12-25\% experiencing distress in relation to it (Palacios 2009). More recent research in other cultures indicates higher rates. A study involving 315 Brazilian-born women in São Paulo, Brazil, found a prevalence of female sexual dysfunction of $35.9 \%$ (Valadares 2008). Rates of various sexual dysfunctions in women in Turkey have also been found to be high. In one Turkish study, the prevalence of female sexual dysfunction, captured by a score on the Female Sexual Function Index (FSFI) of less than 26 , was $43 \%$. Lower educational level, menopause, depression, presence of sexual dysfunction in their partner and contraceptive use were found to be significantly associated with low scores on the FSFI (Aslan 2008). In another Turkish sample (Dogan 2009), the most common female sexual dysfunction was vaginismus (75.9\%), followed by hypoactive sexual desire (9.2\%). Dyspareunia and orgasmic disorder were reported as common comorbid conditions.

As regards men, Nicolosi et al (2003) reported prevalences of erectile dysfunction of $17 \%$ in Italy and 15\% in Brazil.

\section{Africa}

Single-country studies have established only that particular sexual problems are common in certain settings. In primary care samples, age-adjusted rates of erectile dysfunction were found to be 43.4\% in south-west Nigeria (Fatusi 2003), 13.2\% in Ismailia province, Egypt (Seyam 2003), and $54 \%$ in Casablanca, Morocco (Berrada 2003). High rates of sexual dysfunction have also been reported in a series of studies from Kumasi, Ghana. In a community survey of 200 couples, the prevalence of impotence was $60.9 \%$ and of premature ejaculation 65.4\% (Amidu 2011). Prevalences of female sexual problems were also very high, with vaginismus and anorgasmia at $69.3 \%$ and $74.9 \%$ respectively. In another study from Kumasi, 300 sexually active heterosexual males asked about sexual problems: $64.7 \%$ reported premature ejaculation (Amidu 2010a). In females, a prospective survey recorded the prevalence of sexual dysfunction at $72.8 \%$ (Amidu 2010b).

\section{Asia \\ Men}

A cross-national community-based investigation of erectile dysfunction found an age-adjusted prevalence of erectile dysfunction of 34\% in Japan 
and 22\% in Malaysia (Nicolosi 2003). Erectile dysfunction seems to be more prevalent in Asian samples than in European and South American ones. Men from Asian and Islamic backgrounds are also more likely to report premature ejaculation, which again raises questions about what is premature in this context and whether Western norms are being used.

A number of other papers have reported higher rates of sexual dysfunction in Asia across both genders. As regards men, Lewis (2011) analysed 10 studies investigating the prevalence of erectile dysfunction in Asian countries. In general, these countries were observed to have higher rates of erectile dysfunction than comparable European populations. Nicolosi et al (2005) demonstrated large variation in the prevalence rate of erectile dysfunction between Asian countries, with a rate of $2 \%$ reported in Malaysia, compared with 32\% in the Philippines. Other authors have reported even higher rates in Asia, ranging from 9 to $73 \%$ (Ho 2011).

\section{Women}

As regards women, Singh et al (2009) found very high rates of female sexual dysfunction in a medical out-patients clinic in rural South India, notably observing an association between female sexual dysfunction and fewer years spent in education. In this investigation, total scores on the FSFI suggested presence of the disorder in two-thirds (73.2\%) of the 149 women surveyed. The authors point out challenges of conducting this type of study in India, citing sociocultural differences in gender roles and in constructions of both normal sexual functioning and sexual distress. Crosssectional studies of this type identify associations, but developing hypotheses to explain them requires further exploration.

\section{Couples}

In a small case-control study involving Asian and non-Asian couples in the UK, we (Bhugra $\&$ Cordle) attempted to explain differences in demographics of people who presented to a sexual dysfunction clinic in terms of differential pressures to procreate in Asian males (Bhugra 1988). We also pointed to a greater wish in Asian couples for organic rather than psychological explanations for sexual dysfunction (compared with White couples in our clinics).

\section{Study design}

Data on the prevalence of sexual dysfunction in different parts of the world raise important questions in terms of study design and the analysis and interpretation of results. As with any research, a number of questions are important. Why has the study been done, and by whom? What methodological framework was used, and to answer what question? In particular, it is essential to use culturally valid categories for describing illness - applying Western categories to other cultural settings risks committing what Kleinman (1987) calls a category fallacy.

Epidemiological studies of sexual dysfunction are scanty, reflecting several problems, including perhaps secrecy and the low public health importance accorded to the topic, difficulties in conducting well-designed research in this area and unwillingness of individuals to participate in this research. Furthermore, definitions of sexual dysfunctions, and the instruments used to identify and rate them, vary between studies. Populations are community based in many cases, but large multicentre studies with consistent methods of recruitment and questioning have not been done, and the comparability of data is not clear. Nevertheless, evidence appears to point consistently to higher rates of sexual dysfunction in some countries outside the West, and to important variations in the prevalence of sexual dysfunctions within populations. Such distinctions seem meaningful in an increasingly culturally interconnected world.

\section{Ways of thinking about cultural attitudes to sex}

As we have seen, reported prevalence rates of sexual dysfunctions vary in different cultures. Notwithstanding study variables such as sample size, time period over which data were collected, method, outcome measures and many other parameters, it seems reasonable to suppose that there are real, significant differences in the frequency of sexual dysfunctions across cultural groups. How are we to understand this? Differences in rates could be purely related to genetic differences, although this is unlikely given the range of biological, psychological and sociocultural factors that appear to influence sexual functioning.

\section{Sex positivity and sex negativity}

Cultural influences can affect mental disorder through a variety of means. Culture shapes norms of behaviour and sets thresholds for abnormality, and to some degree defines abnormality and deviance. These norms, thresholds and definitions change over time and under the influence of sociopolitical and economic factors. Any consideration of the relationship between culture and sex needs to account for the extremely diverse and shifting theoretical frameworks for both concepts. While 
BOX 2 Sex positivity and sex negativity

In an extensive overview of the role of culture and societies in our understanding of sex, Bullough (1976) defined two broad categories of society in this respect:

- sex-negative societies, which view sex as fundamentally procreative and reject a wider hedonistic or recreational role for sexual activity

- sex-positive cultures, which view sex as more than a purely procreative act.

acknowledging the undoubted complexities of culture, our understanding can only be aided by straightforward, sensible models of this relationship. Sex positivity and negativity (Box 2) offer one such framework within which to summarise cultural attitudes towards sex.

Using these broad categories, the cultural backgrounds of participants (both clinicians and individual patients or couples) may be positioned for the purpose of formulation. However, in terms of conceptualising sexual dysfunction and managing specific cases, this arrangement may be simplistic. For example, sex-negative and sex-positive attitudes may vary depending on circumstances: having done their duty by the society by producing the expected number of children, a couple may turn to sex for intimacy and pleasure. A dichotomous categorisation, or even a spectrum of positivity/ negativity, implies that attitudes towards sexual activity are internally consistent, which seems not to be the case. In addition, it is worth remembering that not all individuals in a sex-positive culture will have positive attitudes to sexual activity, just as not all from sex-negative cultures will have negative views. Sexual attitudes vary considerably within populations, for example in subcultures based on age group or political orientation.

\section{Attitude divergence}

Perhaps an alternative to Bullough's model is to substitute for positivity and negativity an indicator of 'attitude divergence' within a given population.

Another aspect not included in this model is the importance of the way the individual or couple interacts with the prevailing cultural beliefs in their population. Degree of acculturation seems to be a key potential determinant in formulating sexual dysfunction, for example in Asian couples in the UK (Bhugra 1986, 1988), although more research is required in this area. Cultural beliefs in a given population are undergoing constant shift in response to historical and political circumstances, in ways that are not immediately recognisable at the time. Furthermore, there may be room for configurations of sexual attitudes that make more sense in light of globalisation and the increasing confluence of cultural beliefs between places (Mastrogianni 2003). Overall, the influences on sexual function of acculturative stresses and divergence in sexual attitudes within populations remain under-researched. These factors are of interest not only to anthropologists and ethnographers - they may also play an important role in the presentation of sexual problems in the clinic.

\section{Cultural influences on psychopathology}

Tseng (2001) categorises the influence of culture on mental disorder into six possible 'mechanisms':

- pathogenic

- pathoplastic

- pathoselective

- pathofacilitative

- pathoelaborative

- pathoreactive.

These mechanisms may be understood in terms of their relation to the prevailing sex positivity/ negativity of the group in question.

\section{Pathogenic influence}

In directly causing culture-specific conditions/ illnesses, culture plays a pathogenic role. Cultural beliefs can cause stress and emotional distress that are expressed in characteristic ways. For example, in cultures in which it is important to have a son to carry on the lineage, it is inevitable that failure to produce a male child will cause considerable stress. This can give rise to conditions such as dhat, also known as semen-loss anxiety (Sumathipala 2004; Bhugra 2007). In dhat, there is preoccupation that semen is being lost in the urine, and that this confers infertility on the sufferer. Often the symptom is attributed to previous misfortune, and presents alongside strong feelings of self-blame and guilt. However, although the concept of dhat as a syndrome with distinct pathological correlates gained particular prominence during the growth of transcultural psychiatry in the 1960s to 1980s (Yap 1974; Murphy 1977), more recent scholarship has introduced doubt that the syndrome is localised to only one particular cultural setting. In a review of the topic, Sumathipala et al (2004) point out the wider prevalence of semen-loss anxiety in different populations, indicating that this particular syndrome, alongside others, is perhaps not as bound to culture as was previously thought.

\section{Pathoplastic influence}

Culture-bound syndromes with sexual content, of which several are described in the literature, 
are the most direct way in which culture can be seen to influence sexual dysfunction. However, cultural formulations of sexual presentations in clinical work must be viewed in the context of other disorders that may be presenting. For example, authors have commented on the role of dhat as a somatic presentation in depressive illness (Mumford 1996). Here, cultural background is understood to be shaping the particular mode of presentation of the patient experiencing distress, rather than causing the disorder itself - an example of pathoplastic influences.

\section{Pathoselective influence}

The pathoselective role of a culture can be seen when an individual responds to stress in a culturally acceptable way, or when a culture determines acceptable reactions. For example, in certain cultures loss of desire may be a more culturally safe mode of presenting with illness than ejaculatory dysfunction, even if both symptoms are present. In epidemics of koro (genital retraction) syndrome, prevailing cultural beliefs may result in the development of symptoms to generate a sense of belonging. The enduring concept of a sexual syndrome that is culture-bound thereby becomes 'unbound' and becomes assimilated into universal models of somatisation of distress. We (D.B. and coauthors) have argued that the decultured concept of semen-loss anxiety should be analysed in different cultural contexts in order to form a better understanding and management of the condition in different situations. For example, the changing prevalence of semen-loss anxiety in particular groups may be attributed to processes of modernisation, which may be at different stages in different cultural settings, thereby influencing the likelihood of experiencing what may be a universal human condition (Bhugra 2007).

\section{Pathofacilitative influence}

Personal attitudes to sex, for example seeing it as unclean, may influence the attribution of sexual dysfunction as either psychological or somatic. Cultures can also have a pathofacilitative effect, producing certain psychiatric and behavioural conditions. Factors such as the acceptance of a disorder by society, the availability of support and the nature of the existing care system can all provide a foundation on which a condition can develop.

\section{Pathoelaborative influence}

Cultures may also accentuate symptoms in a pathoelaborative way, whereby even universal behaviours are exaggerated to the point of rendering them a disorder. This might affect, for example, concepts of premature ejaculation.

\section{Pathoreactive influence}

In a pathoreactive relationship, culture influences the explanatory models and beliefs related to a particular condition. Culture affects the way in which individuals label a disorder, how they react to it emotionally and seek help, and what resources they use. For example, depression or anxiety may be formulated as occurring as a result of dhat.

\section{Coping and help-seeking}

The explanatory models employed by patients and clinicians are thus very strongly influenced by culture, which may dictate what key words are used to conceptualise distress.

Although research in this area is limited, it may be speculated that relatively restrictive, 'sex-negative' attitudes in a cultural group would be less conducive to effective coping and helpseeking.

As with other aspects of cultural behaviour, an individual's locus of control may be an important explanatory concept in formulations. The locus of control, a concept due to Rotter in the 1950s, is the individual's perceived ability to affect the world around them (internal locus), as opposed to being affected by it (external locus). Individuals with a more externalised locus of control, for example, may be reluctant to recruit and engage the partner in the treatment of sexual problems and may be affected by wider systemic problems within the family. In Asian families, this could be seen as counterintuitive: typically, British Asians form larger, more robust families, which could be seen as more available sources of social support in times of stress. Little is known about alternative pathways of help-seeking for sexual dysfunction among Asians, but observable differences have been found between UK Asian and White couples in points of access to professionals (Bhugra 1988). Divergence in acculturation within a family network (where one or more members may be more acculturated than others) will also shape coping styles and mode of accessing services.

It is important to appreciate that individuals may not share their clinician's understanding of the relationship between mind and body, and between biological sex and gender roles. An understanding of cultural influences based on sex positivity and sex negativity perhaps plays down the role of other salient concepts, such as gender, fertility and the mind-body relationship, that may be useful in understanding sexual dysfunction in unfamiliar cultural contexts. 


\section{Gender roles and sexual dysfunction}

As gender is a social concept, gender roles vary between cultures to some degree. Margaret Mead (1935) was probably the first to suggest that definitions of maleness and femaleness vary crossculturally. It would appear that there are some cultural environments in which greater variability in gender role between individuals is tolerated. These cultures probably also contain more heterogeneity in sexual preference and conduct and greater divergence in sex positivity-negativity. The increasingly globalised cultural milieu has allowed for a change in the definitions and expectations of masculinity and femininity, and has led to a wider range of tolerated behaviours. The implications of the permissiveness of a society for the study of sexual dysfunction are many: it may shape the definitions of sexual dysfunction, modify the threshold at which help is sought and determine the ease of accessing help.

\section{Fertility}

As noted above, a potential consequence of a sex-negative culture is the prominence given to procreation as the primary function of sex. In this way, sex may be seen as intrinsically and exclusively linked to fertility, and notions of sexual dysfunction may be shaped by this relation. Pressure for fertility may be a cause of distress and stress, and can be viewed in both a pathofacilitative (e.g. greater expectations of fertility and procreation in a culture alter the threshold for presenting with conception problems) and a pathoselective (e.g. a couple deciding to present with conception difficulties rather than erectile or orgasmic dysfunction) way. For example, individuals may prefer to articulate dysfunction in terms of problems in conceiving, rather than in simple terms of sexual performance itself.

\section{Mind and body in sexual dysfunction}

Sex is a complex psychophysiological process, and the role of cultural factors, and how they relate to biological variables in all parts of this process, is poorly understood (Bancroft 2002). Psychological stress alters the regularity of the ovulatory cycle and sexual interest (Herbert 1996). Cultural learning over the life course shapes the behavioural patterns associated with sexual arousal. In this respect, sexual responses and processes are experienced differently between cultures, in terms of both the types of symptoms and the meanings they are given (Lock 1998).

Culture may affect the way in which a problem is located by the sufferer, both in terms of the individual $v$. the couple $v$. the family, and in terms of the mind $v$. the body. As Kleinman (1980) has pointed out, somatic symptoms in depression and other emotional problems are more common outside the West, and this observation is paralleled in the study of sexual dysfunction. Malhotra $\&$ Wig (1975) describe a deeply rooted belief among South Asian males that it takes 40 drops of foods to make one drop of fat, 40 drops of fat to make one drop of blood, 40 drops of blood to make one drop of marrow, and 40 drops of marrow to produce one drop of semen. Consequently, masturbation affects both mind and body connectedly (Bhugra 1989).

\section{The individual $v$, the couple}

Western biomedicine positions the individual as central to aetiological theories, although in the assessment of sexual dysfunction, formulations often involve the location of problems within two individuals - the couple. This focus has various shortcomings when it comes to understanding the broader social and cultural implications of illness. Non-Western couples may be non-egocentric. For example, Chinese individuals often present to services within a detailed interpersonal family context, which can introduce complexities for a Western clinician seeking to formulate the case in terms of the couple. In light of the cultural relativities discussed above, couples from different ethnic groups may be even more challenging in this regard, given the possibility that widely differing expectations of sex may be engendered.

\section{Culture, emotion and sexual dysfunction}

The influence of culture on emotions is, not surprisingly, complex. In psychiatry, the characteristics of family communication have been hypothesised to mediate cultural differences in psychopathology. Expressed emotion - the 'amount' and 'temperature' of emotion expressed by an individual in an interpersonal context was initially investigated as a risk actor for the development and relapse of psychotic illness (Vaughn 1981). Patterns of emotional expression have been investigated more recently in eating disorders and attention-deficit hyperactivity disorder (Cartwright 2011; Hoste 2012). The role of expressed emotion in the sexual functioning of couples is not yet clear. However, Lau et al (2005) observed that sexual dysfunction was more common among individuals who shared sleeping quarters with non-spouse family members. The authors suggest that this finding demonstrates the importance of privacy in maintaining sexual functioning; one could also speculate that sharing sleeping quarters with non-spouse family 
members might lead more easily to an emotionally overinvolved family context. These findings point towards a role for family structure in influencing sexual functioning, and should lay the ground for further work, for example to investigate the role of expressed emotion as a mediator of these effects.

\section{Conclusions}

It is clear that certain parts of the non-Western world have much higher rates of some forms of sexual dysfunction than the West, that these variations probably relate to cultural differences, and that these observations should lead to a careful consideration of the role of culture in both the management of sexual dysfunction in different cultural contexts and the design and resourcing of services. However, valid comparisons between populations require further study.

It is important to be aware of cultural influences on sexual behaviour. Laws, power relationships in terms of sex and gender, levels of stress and economic resources are all of significance. Tseng's framework gives us a way of understanding the aetiology and management of sexual dysfunction according to cultural beliefs and values. Incorporating this understanding into formulations is a useful way of articulating and separating risk and protective factors in a given presentation. Furthermore, the importance of cultural factors in shaping definitions and thresholds for sexual difficulties for both the clinician/observer and the patient/participant cannot be underestimated. Expectations of treatment and a detailed positioning of the problem in a social and familial framework are crucial, as is an understanding of the values of clinicians themselves. Such structures are also useful in thinking about the rejection of help in different situations.

More work is obviously necessary on how cultural factors shape the thresholds and definitions of sexual dysfunctions in different groups, and how those considerations may influence management. Future research must carefully identify prevalence of sexual dysfunction in different cultural groups.

There must be a greater focus on modes of presentation between groups. Qualitative methods should be employed to understand the population's own perspectives on their difficulties, the causes of those difficulties and appropriate treatment. Useful formulations depend on clear understanding of the resources available to each patient and group within a population. Individuals may not share the professional's attribution of their problems as cultural and this also needs to be taken into account.

\section{References}

d'Ardenne P (1986) Sexual dysfunction in a transcultural setting: assessment, treatment and research. Sexual and Marital Therapy 1: 23-34.

Amidu N, Owiredu WK, Woode E, et al (2010a) Prevalence of male sexual dysfunction among Ghanaian populace: myth or reality? International Journal of Impotence Research 22: 337-42.

Amidu N, Owiredu WK, Woode E, et al (2010b) Incidence of sexual dysfunction: a prospective survey in Ghanaian females. Reproductive Biology and Endocrinology 1: 106.

Amidu N, Owiredu WK, Gyasi-Sarpong CK, et al (2011) Sexual dysfunction among married couples living in Kumasi metropolis, Ghana. BMC Urology 2: 3 .

Aslan E, Beji NK, Gungor I, et al (2008) Prevalence and risk factors for low sexual function in women: a study of 1,009 women in an outpatient clinic of a university hospital in Istanbul. Journal of Sexual Medicine 5: 2044-52.

Bancroft $J$ (2002) Biological factors in human sexuality. Journal of Sex Research 39: 15-21.

Barnard A, Spencer J (2002) Encyclopaedia of Social and Cultural Anthropology. Routledge.

Berrada S, Kadri N, Mechakra-Tahiri S, et al (2003) Prevalence of erectile dysfunction and its correlates: a population-based study in Morocco. International Journal of Impotence Research 15 (suppl 1): S3-7.

Bullough VL (1976) Sexual Variance in Society and History. John Wiley \& Sons.

Bhugra D, Cordle C (1986) Sexual dysfunction in Asian couples. BMJ 292: 111-2.

Bhugra D, Cordle C (1988) A case control study of sexual dysfunction in Asian and non-Asian couples 1981-1985. Sexual and Marital Therapy 3: $71-6$.

Bhugra D, Buchanan A (1989) Impotence in ancient Indian texts. Sexual and Marital Therapy 4: 87-92.

Bhugra D, Sumathipala A, Siribaddana S (2007) Culture-bound syndromes: a re-evaluation. In Textbook of Cultural Psychiatry (eds D Bhugra, K Bhui): 141-56. Cambridge Medicine.

Bhugra D, Colombini G (2013) Sexual dysfunction: classification and assessment. Advances in Psychiatric Treatment 19: 48-55.

Cartwright KL, Bitsakou P, Daley D, et al (2011) Disentangling child and family influences on maternal expressed emotion toward children with attention-deficit/hyperactivity disorder. Journal of the American Academy of Child and Adolescent Psychiatry 50: 1042-53.

Davis DL (1998) The sexual and gender identity disorders. Transcultural Psychiatry 35: 401-12.

Dogan S (2009) Vaginismus and accompanying sexual dysfunctions in a Turkish clinical sample. Journal of Sexual Medicine 6: 184-92.

Fatusi A0, ljadunola KT, Ojofeitimi EO, et al (2003) Assessment of andropause awareness and erectile dysfunction among married men in Ile-Ife, Nigeria. Aging Male 6: 79-85.

Goettsch S (1987) Textbook sexual inadequacy? A review of sexuality texts. Teaching Sociology 15: 324-38.

Griffitt W, Hatfield E (1985) Human Sexual Behaviour. Scott, Foresman $\&$ Co.

Herbert J (1996) Sexuality, stress, and the chemical architecture of the brain. Annual Review of Sex Research 7: 1-43.

Ho CC, Singam P, Hong GE, et al (2011) Male sexual dysfunction in Asia. Asian Journal of Andrology 13: 537-42.

Hofstede G (2001) Culture's Consequences: Comparing Values, Behaviors, Institutions and Organizations across Nations. Sage.

Hoste RR, Labuschagne Z, Lock J, et al (2012) Cultural variability in Expressed Emotion among families of adolescents with anorexia nervosa. International Journal of Eating Disorders 45: 142-5.
MCO answers

1 a $\quad 2$ a $\quad 3$ b $\quad 4$ c $\quad 5$ a 
Kleinman A (1980) The cultural construction of illness experience and behaviour. In Patients and Healers in the Context of Culture: An Exploration of the Borderland between Anthropology, Medicine, and Psychiatry (Vol. 3). University of California Press.

Kleinman A (1987) Anthropology and psychiatry: the role of culture in cross-cultural research on illness. British Journal of Psychiatry 151: $447-54$.

Lau JTF, Cheng Y, Wang 0, et al (2005) Prevalence and correlates of sexual dysfunction among young adult married women in rural China: a population-based study. International Journal of Impotence Research 18: 89-97.

Lewis RW (2011) Epidemiology of sexual dysfunction in Asia compared to the rest of the world. Asian Journal of Andrology 13: 152-8.

Lock M (1998) Menopause: lessons from anthropology. Psychosomatic Medicine 60: 410-9.

Malhotra HK, Wig NN (1975) Dhat syndrome: a culture bound sex neurosis of the Orient. Archives of Sexual Behaviour 4: 519-28.

Masters WH, Johnson VE (1970) Human Sexual Inadequacy. Bantam Books.

Mastrogianni A, Bhugra D (2003) Globalization, cultural psychiatry and mental distress. International Journal of Social Psychiatry 49: 163-5.

Mead M (1935) Sex and Temperament in Three Primitive Societies. Reprinted 2001, Harper Perennial.

Mumford DB (1996) The 'Dhat syndrome': a culturally determined symptom of depression? Acta Psychiatrica Scandinavica. 94: 163-7.

Murphy HBM (1977) Migration, culture and mental health. Psychological Medicine 7: 677-84.

Nicolosi A, Moreira Jr ED, Shirai M, et al (2003) Epidemiology of erectile dysfunction in four countries: cross-national study of the prevalence and correlates of erectile dysfunction. Urology 61: 201-6.

Nicolosi A, Glasser DB, Kim SC, et al (2005) Sexual behaviour and dysfunction and help-seeking patterns in adults aged $40-80$ years in the urban population of Asian countries. British Journal of Urology International 95: 609-14.

Palacios S, Castaño R, Grazziotin A (2009) Epidemiology of female sexual dysfunction. Maturitas 63: 119-23.

Prince M, Stewart R, Ford T, et al (eds) (2003) Practical Psychiatric Epidemiology. Oxford University Press.

Reiss IL (1986) Journey into Sexuality. Little Brown

Seyam RM, Albakry A, Ghobish A, et al (2003) Prevalence of erectile dysfunction and its correlates in Egypt: a community-based study. International Journal of Impotence Research 15: 237-45.

Shifren JL, Monz BU, Russo PA, et al (2008) Sexual problems and distress in United States women: prevalence and correlates. Obstetrics \& Gynecology 112: 970-8.

Singh K (1960) Psychosexual disorder in the male: a study of 96 cases. Indian Journal of Psychiatry 12: 100-5

Singh JC, Tharyan P, Kekre NS, et al (2009) Prevalence and risk factors for female sexual dysfunction in women attending a medical clinic in south India. Journal of Postgraduate Medicine 55: 113-20.

Sumathipala A, Siribaddana SH, Bhugra D (2004) Culture-bound syndromes: the story of dhat syndrome. British Journal of Psychiatry 184: $200-9$

Tseng WS (2001) Handbook of Cultural Psychiatry. Academic Press.

Valadares AL, Pinto-Neto AM, Osis MJ, et al (2008) Prevalence of sexual dysfunction and its associated factors in women aged 40-65 years with 11 years or more of formal education: a population-based household survey. Clinics (Sao Paulo) 63: 775-82.

Vaughn CE, Leff JP (1981) Patterns of emotional response in relatives of schizophrenic patients. Schizophrenia Bulletin 7: 43-4.

Weis DL (1998) Conclusion: the state of sexual theory. Journal of Sex Research 35: 100-14.

Yap P (1974) Comparative Psychiatry: A Theoretical Framework. University of Toronto Press.

\section{MCOs}

Select the single best option for each question stem

1 The notion that individuals vary in the way they attribute events to internal and external explanations was developed by:

a Rotter

b Kleinman

c Masters

d Johnson

e Rutter.

\section{Variations in sexual arousal between} populations is most likely to relate to:

a cultural differences

b historical differences

c genetic differences

d environmental differences

e chance.
3 Which of the following approaches is most closely identified with Masters \& Johnson?

a sex-positive and sex-negative cultures

b sensate focus

c locus of contro

d expressed emotion

e individualism $v$. collectivism.

4 Which of the following is an appropriate measure of sexual dysfunction among English speakers?

a Hospital Anxiety and Depression Scale (HADS)

b Montgomery-Åsberg Depression Rating Scale (MADRS)

c Female Sexual Function Index (FSFI)

d Yale-Brown Obsessive Compulsive Scale (YBOCS)

e Abnormal Involuntary Movement Scale (AIMS).
5 Which of the following 'culture-bound syndromes' presents with sexual difficulties?

a koro

b ataque de nervios

c amok

d latah

e brain fag. 